\title{
An Overview of Immunologic Adjuvants - A Review
}

\section{Aiyer Harini P*, Ashok Kumar HG, Gupta Praveen Kumar and Shivakumar Neeta}

Department of Biotechnology, R. V College of Engineering, Bangalore-59, India

\begin{abstract}
With the onset of antigen production using biosynthetic and rDNA techniques, the need to administer adjuvants along with vaccines has increased remarkably. This search for accompanying adjuvants has resulted in a multitude of molecules from mineral salts to bacterial polysaccharides and Immuno stimulatory complexes that range considerably in efficacy. Moreover, the adjuvant molecule is specific to its antigen molecule and hence has to be tailored suitably to maximise efficacy and safety, maintaining the cost at a minimum. This paper explores the characteristics of these molecules, their mode of action and the advancements in the field.
\end{abstract}

Keywords: Adjuvant; Immunogen; Recombinant DNA technology; Saponins

\section{Introduction}

The word adjuvant is derived from the Latin word adiuvare; meaning to help or aid, refers to any material that enhances the cellular or humoral response to an antigen. They comprise a diverse group of defined molecules or more complex formulations [1] and have been used since the early $20^{\text {th }}$ century to help improve this response. The need for adjuvants has arisen because many vaccines produce a poor immunological response on their own.

\section{Need for Adjuvants}

Vaccines, one of the most successful medical inventions against various infectious diseases, (Hillman), sometimes require a molecule in conjugation that augments its immune response. Previously, antibodies used to be made in response to proteins, carbohydrates, complex lipids and nucleic acids isolated from natural sources. However, the antigens today, are made using modern chemical, biosynthetic and rDNA techniques; a majority of which are weak immunogens due to the lack of an innate immune stimulus [2,3]. Small polypeptides $(<10 \mathrm{kDa})$ and nonprotein antigens need to be conjugated to a large immunogenic carrier protein to become good immunogens. It is therefore expedient to co-administer these with an adjuvant to ensure a high quality/high quantity, memory-enhanced antibody response.

\section{Adjuvants can be subjected to various uses such as}

1. To bolster the immune response of any antigens by delivering in native form.

2. To reduce the multiple immunization protocol for protective immunity. In particular to develop single step vaccination coverage that can reduce the vaccination costs.

3. To enhance the immune response of immune compromised adults and weakened immune system of children, to elicit cytotoxic $\mathrm{T}$ lymphocytes response and generate local immune response [4].

\section{Classification of Adjuvants}

The adjuvant property of a molecule increases with the length of the sugar side chain and the HLB value have high hydrophile-lipophile balance (HLB) value [5]. However, adjuvants are conventionally classified into the following categories: Mineral compounds, Bacterial products, Oil-based emulsions, ISCOMs and Liposomes. Of these, the aluminium based mineral compounds are the most widespread [6] and the most preferred for humans [7] (Figure 1).

\section{Aluminium based minerals}

Aluminium based adjuvants, like Aluminium hydroxide and

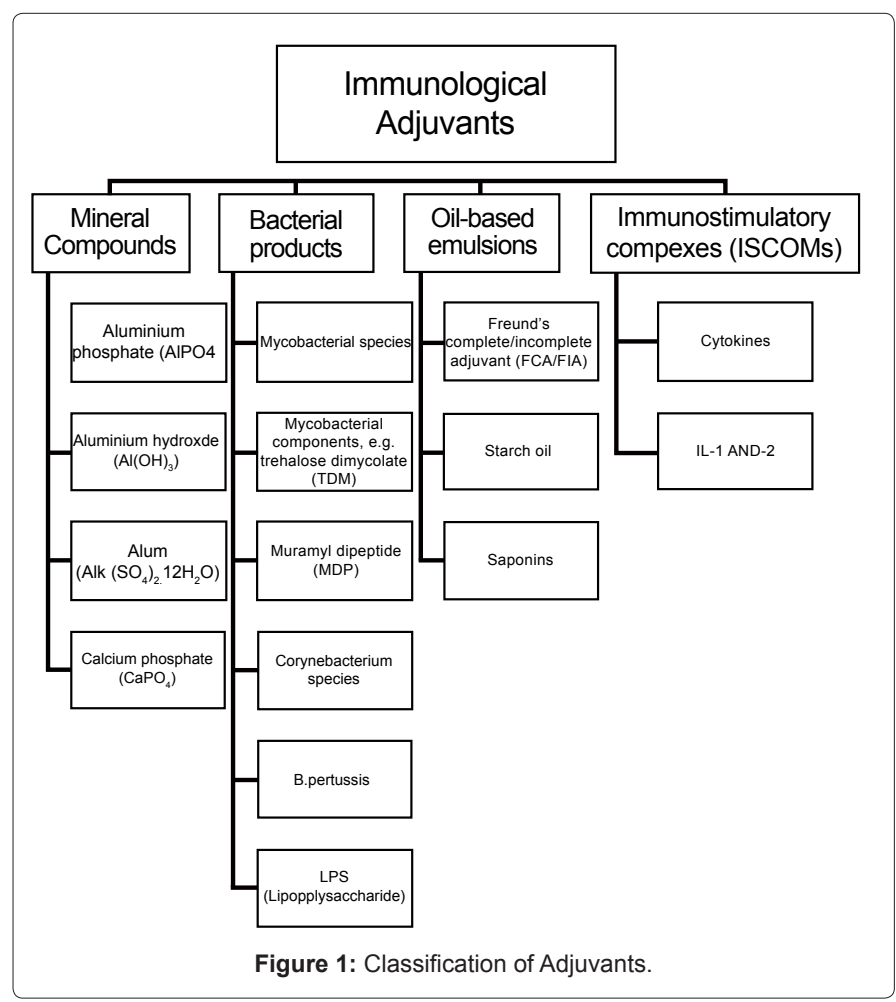

*Corresponding author: Aiyer Harini P, Department of Biotechnology, R.V College of Engineering, Bangalore-59, India, Tel: +91- 9035426543; E-mail: harini.p.aiyer@gmail.com

Received December 19, 2012; Accepted January 27, 2013; Published January 29, 2013

Citation: Aiyer Harini P, Ashok Kumar HG, Praveen Kumar G, Shivakumar N (2013) An Overview of Immunologic Adjuvants - A Review. J Vaccines Vaccin 4 167. doi:10.4172/2157-7560.1000167

Copyright: (C) 2013 Aiyer Harini P, et al. This is an open-access article distributed under the terms of the Creative Commons Attribution License, which permits unrestricted use, distribution, and reproduction in any medium, provided the original author and source are credited. 
Aluminium phosphate have been known to induce early, long lasting, high titre, protective immunity [5]. However, aluminium is a weak adjuvant for antibody induction to recombinant protein vaccines [8] (Figure 2).

\section{Oil-based emulsions}

These are popular immune potentiators for inactivated vaccines [7].

Saponins: Saponins are steroid or triterpenoid glycosides, which occur in many plant species, in both wild plants and cultivated crops. In cultivated crops the triterpenoid saponins are generally predominant, whereas steroid saponins are common in plants used as herbs or for their health-promoting properties. Saponin-based adjuvants have the unique ability to stimulate cell-mediated immunity, as well as to enhance antibody production [7]. Research on certain traditional Chinese medicinal herbs such as Panax ginseng, Astragalus species, Panax notoginseng have gained attention as candidates for plant derived saponins [3]. Quillaja saponaria extract as adjuvants, first described in the 1930s have been the most prominent of the saponins used as adjuvants to feature in the respective literature $[9,10]$ (Figure 3).

\section{Bacterial products}

Due to their potent immunostimulatory capacity, bacterial products are considered a good source of immunological adjuvants. Bacterial flagellin is an effective adjuvant for CD4+ T cells in vivo [11].

Heat shock proteins (HSPs) are conserved proteins that are highly immunogenic and function as adjuvants that may play a crucial role in integrating innate and adaptive immunity [12].

\section{Cytokines}

Cytokines like IFNg or GM-CSF have been popular for over a decade as effective adjuvant molecules [13]. Induction of local delayed hypersensitivity (DTH) is commonly observed after the use of Proinflammatory cytokines IL-1, TNF- , IFN- , IFN- , IL-6, IL-8 [14].

\section{Selection of Adjuvants}

Immunological adjuvants accelerate, prolong or enhance antigen-

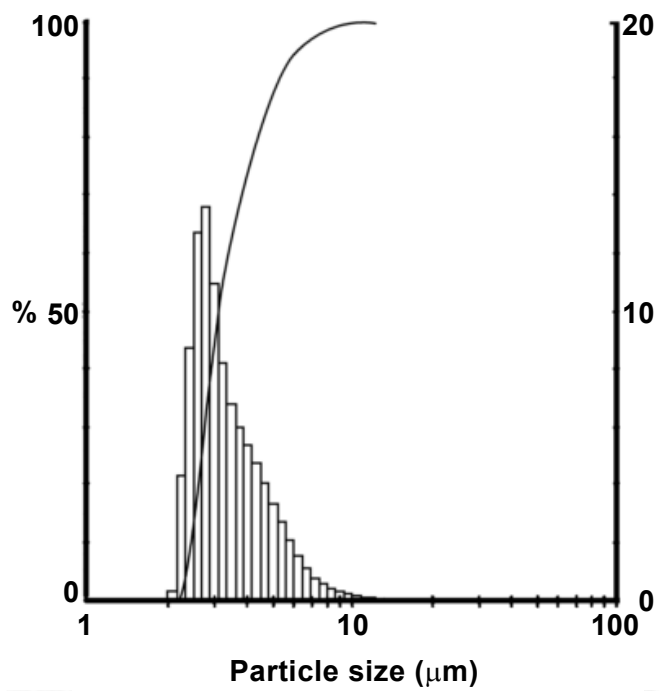

Figure 2: Typical particle size distribution of Aluminium hydroxide adjuvant (Alhydrogel) [5]
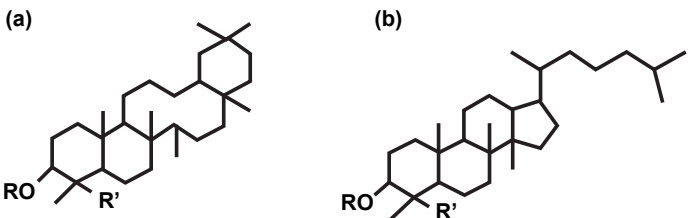

Figure 3: Basic structures of sapogenins: a triterpenoid (a) and a steroid (b) [10].

specific immune responses if used in combination with specific vaccine antigens. Ideally an adjuvant is assumed to possess long shelf life with undiminishing stability, biodegradability, low cost of production, the ability to not induce immune responses against itself and to promote the required immune response. However, observations have been made of differences in adjuvant efficacy with the route of administratione.g. between mucosal and parenteral routes. Therefore, the adjuvant should be selected by considering the various factors involved [2]. For example, it was discovered that subunit vaccine responses can be enhanced relative to soluble antigen/adjuvant or alum formulations [15]. Kreuter and Haenzel [16] observed that the particle size of the polymer adjuvant was found to be an important parameter for adjuvant activity.

\section{Mode of Action of Adjuvants}

Vaccines based on highly purified antigens will require specific adjuvants to elicit the required response [1]. Targeting of vaccines to specific immune cells is very promising. However, it may be difficult to develop effective vaccines without blocking immune regulatory pathways thus hampering the CMI response. Adjuvants have significant effects on the immune responses, and can tip the immune system in favour of Th1 or Th2 type response [9].

To sustain an Ab response, a supply of Ag is needed. One way an adjuvant may aid the immune response is by forming a depot of $\mathrm{Ag}$ at the injection site resulting in the sustained release of small quantities of Ag over a long period of time. Even with an adjuvant that forms a depot of $\mathrm{Ag}$, at some point in time the quantity of $\mathrm{Ag}$ is diminished and the $\mathrm{Ab}$ titer declines. At this time a second injection of Ag (a booster dose) may be given. When an animal that has responded maximally is given a booster dose of $\mathrm{Ag}$ too soon, suppression rather than enhancement of the immune response may ensue.

Alternatively, an adjuvant can work is to serve as a vehicle to help deliver the Ag to the spleen and/or lymph nodes where Ag is trapped by the follicular dendritic cells and where most of the necessary cell to cell interactions take place to generate plasma cells (the Ab-secreting cells). For example, microdroplets of oil containing $\mathrm{Ag}$, such as those formed in an oil-in-water adjuvant emulsion, are readily ingested by macrophage and taken to draining lymph nodes or spleen. Ag-loaded tissue dendritic cells rapidly emigrate via lymphatics to draining lymph nodes. Additionally, emulsions aid tissue dendritic cells in their capture of Ag.

A third way an adjuvant can work is to activate the various cells involved in the immune response, either directly or indirectly. Surfactants, components of all emulsion adjuvants, may serve this function as well as helping to stabilize oil-water emulsions. Also, many bacteria contain substances that activate cells of the immune system, particularly the macrophage. The activated macrophage in turn helps activate $\mathrm{T}$ and $\mathrm{B}$ cells. Thus some adjuvants contain bacteria, bacterial products, or derivatives of bacterial products. Although the activation of 
macrophages indeed aids in the antibody response, excessive activation of macrophages also causes excessive inflammation, so that bacterial components cannot be used in excess. In recent years, a number of bacterial products have been modified in ways that maximize their desirable activation potential and minimize their inflammatory potential with the goal of finding ideal adjuvant components. For example, some of the new generation adjuvants incorporate a chemical variant of endotoxin called monophosphoryl lipid A [MPL] or a modified muramyl dipeptide [thr-MDP] or other "detoxified" cell wall constituents of bacteria [17]

\section{Advancements}

Adjuvant formulations can be tailored to enhance the required immune response (antibody, cell mediated, mucosal immunity) specific to individual causative infectious agents [18].

The Matrix Immune Modulator (MIM) was developed to overcome real and perceived disadvantages of classical mineral salt adjuvants. It not only potentiated the immune response to antigens but also increased antibody production in chickens and mice, thus suggesting MIMs as a potential substitute for mineral based adjuvants [19].

Baldwina et al. [20] compared a stable oil-in-water emulsion (SE) and a stable oil-in-water emulsion incorporating glucopyranosyl lipid adjuvant, a synthetic TLR-4 agonist (GLA-SE), each together with a recombinant protein, ID93. Their study highlighted the emphasis on administering effective adjuvants along with the subunit vaccines for treatment against tuberculosis.

BAE, or biologically active molecules purified from a Brazilian palm-tree fruit- the babassu, have been shown to possess potential adjuvant properties. Research suggests that it could be administered in association with or without aluminium compounds, for the preferential induction of Th1-dependent immune responses against different antigens in distinct murine strains and animal species [20].

Adjuvants are being studied for the treatment of cutaneous melanoma. Oncogenic BRAF inhibitors such as vemurafenib have been proposed to be used in the adjuvant setting [21].

The immunologic enhancement mediated by a polysaccharide (PPSB) from the fruits of Physalis alkekengi yielded results which indicated that both humoral immunity and cellular immunity were mediated by the polysaccharide. It is hence a promising adjuvant eliciting both Th1 and Th2 responses to help improve the efficacy of vaccine [20].

\section{Software that Aids Adjuvant Selection}

Adjuvant softwares have been developed to provide an estimate of whether or not to receive systemic adjuvant therapy for cancer depends on weighing the benefit (in terms of overall survival) against the cost and risk associated with such therapy.

The most popular software 'Adjuvant!' uses data from national databases and other sources to estimate a patient's baseline prognoses. It requires an experienced health professional to enter the data on the website and obtain the results and the toxicity review sheets for the adjuvant regimens to be discussed [22] (Figure 4).

\section{The Search for Additional Adjuvants}

Since adjuvants have to be tailor made for the vaccine and due to the tantamount importance on the safety associated with its administration, a search has arisen for adjuvants which are capable of inducing:

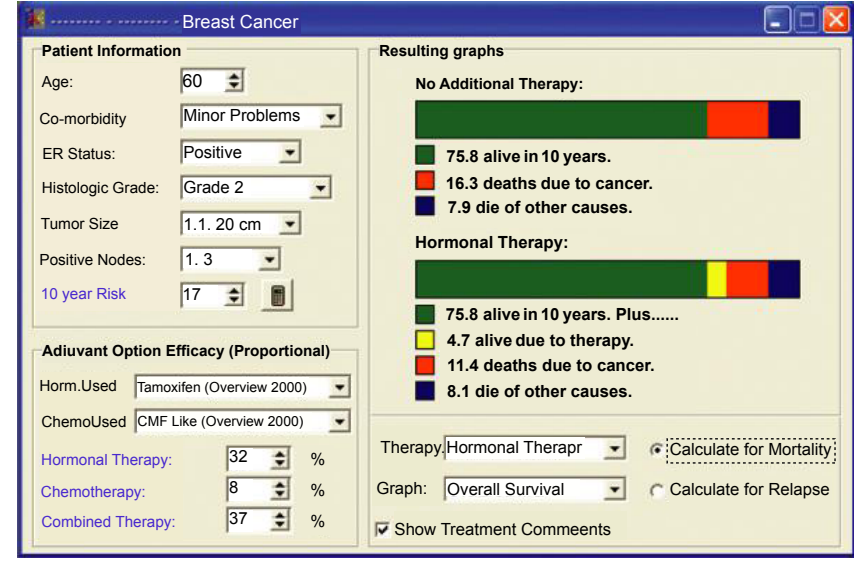

Figure 4: Main Screen of Adjuvant! [23].

1. Broader immune responses covering multiple serotypes.

2. Strong T-cell responses that are needed against infections such as hepatitis $\mathrm{C}$ virus and human immunodeficiency virus (HIV)

3. Responses which stimulate an immune response early in life.

4. Potent mucosal immunity

5. Immune responses to poor immunogenic antigens [23].

Over the last decades very few adjuvants have been licensed for prophylactic vaccines due to toxic properties detected during preclinical or clinical studies (Progress in understanding adjuvant immune toxicity mechanisms Alexander Batista). The advent of recombinant technology in formulation of vaccines has only exalted the demand for adjuvants. Therefore, immense research is required to find a suitable adjuvant for a particular vaccine with maximum safety and efficacy.

\section{References}

1. Schijns VE, Lavelle EC (2011) Trends in vaccine adjuvants. Expert Rev Vaccines 10: 539-550.

2. Petrovsky N, Aguilar JC (2004) Vaccine adjuvants: current state and future trends. Immunol Cell Biol 82: 488-496.

3. Song X, Hu S (2009) Adjuvant activities of saponins from traditional Chinese medicinal herbs. Vaccine 27: 4883-4890.

4. Sivakumar SM, Safhi MM, Kannadasan M, Sukumaran N (2011) Vaccine adjuvants - Current status and prospects on controlled release adjuvancity. Saudi Pharmaceutical Journal 19: 197-206.

5. Lindblad EB (2004) Aluminium compounds for use in vaccines. Immunol Cell Biol 82: 497-505.

6. Cox JC, Coulter AR (1997) Adjuvants--a classification and review of their modes of action. Vaccine 15: 248-256

7. Jansen T, Hofmans MP, Theelen MJ, Manders F, Schijns VE (2006) Structureand oil type-based efficacy of emulsion adjuvants. Vaccine 24: 5400-5405

8. Rajput ZI, Hu SH, Xiao CW, Arijo AG (2007) Adjuvant effects of saponins on animal immune responses. J Zhejiang Univ Sci B 8: 153-161.

9. Sun HX, Xie Y, Ye YP (2009) Advances in saponin-based adjuvants. Vaccine 27: $1787-1796$

10. Francis G, Kerem Z, Makkar HP, Becker K (2002) The biological action of saponins in animal systems: a review. Br J Nutr 88: 587-605.

11. Mcsorley SJ, Ehst BD, Yu Y, Gewirtz AT (2002) Bacterial Flagellin is an effective adjuvant for CD4+ T cells in vivo. J Immunol 169: 3914-3919

12. Ebrahimi SM, Tebianian M (2011) Role of Mycobacterial Heat Shock Protein 70 (mHSP70) as Genetic Vaccine Adjuvants. World Applied Sciences Journal 14: $1569-1575$. 
Citation: Aiyer Harini P, Ashok Kumar HG, Praveen Kumar G, Shivakumar N (2013) An Overview of Immunologic Adjuvants - A Review. J Vaccines Vaccin 4: 167. doi:10.4172/2157-7560.1000167

Page 4 of 4

13. Aucouturier J, Dupuis L, Ganne V (2001) Adjuvants designed for veterinary and human vaccines. Vaccine 19: 2666-2672.

14. Batista-Duharte A, Lindblad EB, Oviedo-Orta E (2011) Progress in understanding adjuvant immunotoxicity mechanisms. Toxicol Lett 203: 97-105.

15. Jewell CM, López SC, Irvine DJ (2011) In situ engineering of the lymph node microenvironment via intranodal injection of adjuvant-releasing polymer particles. Proc Natl Acad Sci U S A 108: 15745-15750.

16. Kreuter J, Haenzel I (1978) Mode of action of immunological adjuvants: some physicochemical factors influencing the effectivity of polyacrylic adjuvants. Infect Immun 19: 667-675.

17. Baker PJ, Hiernaux JR, Fauntleroy MB, Stashak PW, Prescott B, et al. (1988) Ability of monophosphoryl lipid $A$ to augment the antibody response of young mice. Infect Immun 56: 3064-3066.

18. Visciano ML, Tagliamonte M, Tornesello ML, Buonaguro FM, Buonaguro L
(2012) Effects of adjuvants on IgG subclasses elicited by virus-like particles. J Transl Med 10: 4

19. Ritchie RD, Overby A, Suckow MA A Paradigm Shift For Adjuvants: The Matrix Immune Modulator.

20. Baldwina SL, Foxa CB, Pallansch MA, Coler RN, Reed SG, et al. (2011) Increased potency of an inactivated trivalent polio vaccine with oil-in-water emulsions. Vaccine 29: 644-649.

21. Davar D, Tarhini AA, Kirkwood JM (2012) Adjuvant therapy for melanoma Cancer J 18: 192-202.

22. Adjuvant!

23. Mastelic B, Ahmed S, Egan WM, Del Giudice G, Golding H, et al. (2010) Mode of action of adjuvants: implications for vaccine safety and design. Biologicals 38: $594-601$ 